\title{
Generalized likelihood ratio test for optical subpixel objects' detection with hypothesis-dependent background covariance matrix
}

\author{
Victor Golikov $\odot,{ }^{\text {a,* }}$ Oleg Samovarov ${ }^{\mathrm{b}, \mathrm{c}}$ Evgeniy Zhilyakov, ${ }^{\mathrm{d}}$ \\ Jose L. Rullan-Lara, ${ }^{a}$ and Hussain Alazki $\odot^{a}$ \\ ${ }^{a}$ Autonomous University of Carmen, Engineering Faculty, Ciudad del Carmen, Mexico \\ ${ }^{\mathrm{b}}$ Ivannikov Institute for System Programming, Moscow, Russia \\ ${ }^{c}$ Plekhanov Russian University of Economics, Moscow, Russia \\ ${ }^{\mathrm{d} B e l g o r o d ~ S t a t e}$ University, Belgorod, Russia
}

\begin{abstract}
Much interest has arisen in the problem of detecting weak optical subpixel objects in a sequence of images immersed in a heavy homogeneous Gaussian clutter background. In optical systems, the presence of the objects changes the background plus the channel noise covariance matrix. Hence, this matrix may be different under null and alternative hypotheses. Because the maximum likelihood estimate of the background covariance matrix is not available when using the primary data set, we use an approximate estimate of a certain number of eigenvalues of the background covariance matrix that are available for estimation. We derived the general likelihood ratio test for the problem at hand and obtained the modified spectrum matching detector (MSpMD). Using the simulation results, we contrast it with well-known spectrum matching detectors and the hypothesis-independent matched subspace detectors. We also present experimental results of small subpixel objects detection on an agitated sea surface, using digital optical experiments that demonstrate the validation of the simulating results. We show the advantage of the MSpMD that allows to detect small subpixel floating objects on an agitated sea surface even with the same means and variances of the reflected signals from the sea surface and the object. () The Authors. Published by SPIE under a Creative Commons Attribution 4.0 Unported License. Distribution or reproduction of this work in whole or in part requires full attribution of the original publication, including its DOI. [DOI: 10.1117/1.JRS.14.046513]
\end{abstract}

Keywords: subpixel detection; image sequences; unknown background spectra.

Paper 200662 received Sep. 5, 2020; accepted for publication Nov. 17, 2020; published online Dec. 2, 2020.

\section{Introduction}

The detection of optical objects immersed in an observed scene can be difficult when the object is embedded in a dominant cluttered background. Due to the limitations of the spatial resolution of imagery, the objects are often smaller than the pixel size and resolved within a pixel. In such cases, the objects are called subpixel objects. The solution to this problem is very difficult especially when the object area is less than the space resolution of one pixel (subpixel case).

The common detection algorithms include the spectral feature fitting (SFF), ${ }^{1-4}$ orthogonal subspace projection (OSP), ${ }^{5-7}$ constrained energy minimization (CEM) ${ }^{5,8}$ matched filter (MF), ${ }^{1,9-17}$ and mixture tuned matched filtering (MTMF) ${ }^{18-22}$ The SFF method uses a spectral absorption feature in its comparisons. It may fail to extract the object of interest when the object does not have obvious spectral characteristics distinguished from those of the background. OSP requires the spectral signature of both the object and background. CEM is a linear filter, which constrains a desired target signature while minimizing the total energy of the output of other unknown signatures. CEM requires spectral prior knowledge of the object and utilizes the second-order statistical information of the image. MF is another widely used object detection algorithm, which is deduced from the generalized likelihood ratio test (GLRT). ${ }^{9,11}$ In the Bayes or

*Address all correspondence to Victor Golikov, vgolikov@pampano.unacar.mx 
Neyman-Pearson sense, when the object and background classes follow multivariate normal distributions with the same covariance matrix, the MF detector can get an optimum detection result. In fact, MF and CEM detectors have a similar form, and the main difference is that the data used in MF detector have to be centralized first. MTMF ${ }^{17,18}$ combines the statistical method of the MF with the linear mixing model, which can produce a better detection result.

Adaptive cosine/coherent estimate (ACE) is another statistical hypothesis test-based method. ${ }^{3,18}$ Unlike an adaptive matched subspace detector (AMSD), ACE assumes no structured background. Instead, ACE models the background as a multivariate normal distribution. AMSD is an algorithm that formulates the object and background subspaces and uses the linear mixture model and the generalized likelihood ratio (GLR) approach to separate a probable subpixel object. ${ }^{17,18}$ The key factors in the AMSD's design (synthesize) are the object and the background parameters, which can be estimated by the maximum likelihood (ML) method and with which a Neyman-Pearson detector can be designed to maximize the probability of detection at a certain probability of the false alarm.

The matched subspace detector (MSD) is a classical subspace-based method for subpixel target detection. This method has been intensively developed in recent years for solving various applied problems. The recently proposed MSDinter ${ }^{23}$ introduces "interaction effects" terms into the popular MSD from regression analysis in multivariate statistics and the bilinear mixing model in spectral unmixing. The matched shrunken subspace detector ${ }^{24}$ and matched shrunken cone detector ${ }^{25}$ are developed by shrinking the abundance vectors of the target and background subspaces in the hypothesis models of the MSD. The noise model assumes that noise has the same variance over different bands, which is usually unrealistic in practice. In Ref. 26, it was the equal variance assumption and proposed a matched subspace detector with heterogeneous noise (MSDH).

The statistical features of the noise and the background for statistical hypotheses $H_{0}$ and $H_{1}$ may be different from each other. The hypothesis-independent method was introduced to solve this problem, where the noise power is only known under the null hypothesis and the background power depends on statistical hypothesis. Recently, in Ref. 27, using this method, the hypothesisindependent matched subspace detector (HMSD) was obtained.

In optical systems, the small object may completely cover the pixel cell on the fluctuating sea surface, and, in this case, the received signal contains only the target signal plus channel noise. Hence, the presence of the object shades the sea surface and removes the sea clutter from the received signal. When the object does not completely cover the pixel's area, the received signal is equal to sum of the signal reflected from the sea surface plus channel noise and the signal from the object plus channel noise. The covariance matrix of this signal depends on the shape of the background covariance matrix, the area occupied by the object inside the pixel's area, and the ratio of the background power to the channel noise power. Therefore, in this case, it is more appropriate to use the different background covariance matrices for the different hypotheses.

In the literature, the detection problem with the hypothesis-dependent noise power had been first considered in Ref. 12 for the case of the white normal noise. In Ref. 12, Vincent et al. examined specific aspects of the situation where the presence of the signal of interest triggers an increase in the noise power. In Ref. 13, the asymptotic optimum quadratic detector of the subpixel targets in a Gaussian environment has been designed. It was assumed that the background covariance matrix and power under the null hypothesis are a priori known, but under the alternative hypothesis is that the background power can be a priori unknown. The background has the same normalized covariance matrix under hypotheses $H_{0}$ and $H_{1}$, but different variances. In Ref. 13, the method of whitening data is used to design the GLRT.

In this paper, we consider the problem of detecting small subpixel optical floating objects surrounded by an agitated sea surface in the sequence of $N$ digital images immersed in the Gaussian channel noise. We assume that the subpixel object may be present completely or partially anywhere in the pixel's area. We assume that the subpixel area is partially or completely covered by the object, and the presence of the object changes the received signal covariance matrix. In other words, we are considering a case where the appearance of an object changes the dynamic background in the area where it appeared. The common drawback of the published works $^{10-12}$ is the assumption that the background plus noise normalized covariance matrix under 
hypothesis $H_{0}$ remains the same one as under hypothesis $H_{1}$. This case corresponds to the behavior of the reflected signals in radar, sonar, and communication systems. In optical/infrared systems, it is typical that the appearance of the object shades the background, hence the received signal has different covariance structures under hypotheses $H_{0}$ and $H_{1}$. When the different noise covariance matrices correspond to a different statistical hypothesis, it is not possible to use the whitening method for GLRT synthesis. To solve this problem, the singular value decomposition method is used here. In the asymptotic approximation, the eigenfunctions of the noise covariance matrices are orthogonal complex exponential functions. Applying these functions to input signals diagonalizes the noise covariance matrices for both hypotheses. GLRT synthesis requires MLE of the unknown noise parameters. In this case, the MLE corresponds to an estimate of the spectral components of the background. In practice, the width of the spectrum of reflections from the object is less than the width of the background spectrum, which makes it possible to estimate the energy spectrum of the background at an alternative hypothesis in a frequency band free of the useful signal. These spectral estimates are used to obtain the modified spectrum matched detector (MSpMD).

The remainder of this paper is organized as follows. Section 2 describes our modified spectrum matching detector. Section 3 details the simulation experiments for comparative detectors assessments of the proposed detector, SpMD, and HMSD. The results of the real experiments showing that the proposed detector performance is higher than the SpMD and the HMSD are also presented. Section 4 summarizes this paper.

\section{Problem Formulation and Detector Designs}

\subsection{Scene}

We consider a signal at the output of the video camera composed of $K$ successive frames. The typical object is floating and fluctuating according to the sizes of marine waves and its own shape, size, weight, etc. The reflected light signals from a typical object and its spectrum depend on its oscillations on the sea surface. In this paper, we consider the subpixel case when the target is embedded within a single pixel and occupies some part of the pixel's area. Sea surface behavior consists of a superposition of random sinusoidal surface waves and is mainly driven by the wind velocity. The spectral distributions of the reflected light signals from sea surface depend on wind speed (model by Pierson and Moskowitz) and typically contain a quantity of spectral components much more than the number of the floating object spectral components.

\subsection{Background Space and Model}

It is assumed that the optical background clutter is the predominant noise factor and is modeled as a correlated Gaussian noise with covariance matrix $\mathbf{R}_{b}$. The video camera receives $K$ successive frames of the reflected signal plus a channel noise. This paper discusses the detection of a small floating object using a vector $\mathbf{x}=\left[\begin{array}{llll}x_{1} & x_{2} & \cdots & x_{N}\end{array}\right]^{T}$ that consists of the values received from the same pixel in successive frames. It is assumed that the channel noise is uncorrelated white Gaussian noise then background signal $\mathbf{x} \sim N\left(\mathbf{m}, \mathbf{R}_{b n}\right)$. We consider in this paper the stationary homogeneous background where the vector $\mathbf{m}=\left[\begin{array}{llll}m_{1} & m_{2} & \cdots & m_{N}\end{array}\right]^{T}$ is the vector of the average for the any pixel (in the homogeneous case) in the different frames of the background and $m_{1}=$ $m_{2}=\cdots=m_{N}$ in the stationary case. The covariance matrix of the received background signals $\mathbf{R}_{b n}=\mathbf{R}_{b}+\sigma_{n}^{2} \mathbf{I}$. The covariance matrix is symmetric and nonnegative definite. Therefore, there exists an orthogonal matrix $\mathbf{U}$ such that $\mathbf{R}_{b n}=\mathbf{U D}_{b n} \mathbf{U}^{H}$. Here, $\mathbf{U}$ is the $K \times K$ eigenvector matrix of the background plus noise covariance matrix with eigenvalues $\lambda_{0} \geq \lambda_{1} \geq \ldots \geq \lambda_{(N-1)} \geq 0, \mathbf{D}_{b n}=\operatorname{diag}\left[\lambda_{b n 0}^{2} \cdots \lambda_{b n(N-1)}^{2}\right]$. In the stationary case for $N \gg 1$, we assume that the eigenvector matrix $\mathbf{U}$ can be approximated as the basis matrix $\mathbf{H}=\left[\mathbf{h}_{0}, \mathbf{h}_{1}, \ldots, \mathbf{h}_{N-1}\right]$ with orthonormal columns. As such, as $N \rightarrow \infty$, the eigenvalues $\lambda_{i}^{2}$ and eigenvectors $\mathbf{h}_{i}=\frac{1}{\sqrt{K}}\left[h_{i 0} h_{i 1} \cdots h_{i(N-1)}\right]^{T}$ are easily found. ${ }^{14}$ Letting $P_{x}(f)$ denote the power spectral density (PSD) of $\mathbf{x}$ we have that as $N \rightarrow \infty, \lambda_{i}^{2}=P_{x}\left(f_{i}\right), h_{\text {in }}=\exp \left(j 2 \pi n f_{i}\right)$, for 
$i, n=0,1, \ldots,(N-1)$, frequencies $f_{i}=i / N$, and $j=\sqrt{-1}$. The eigenvalues $\lambda_{i}^{2}$ are equally spaced samples of the PSD over the frequency $f_{i}$ interval $[0,1]$ and the eigenvectors $\mathbf{h}_{i}$ are the discrete Fourier transform (DFT) vectors. The approximation will be a good one where the data record length $N$ is much larger than the correlation time of $\mathbf{x}$. Note that the vectors $\mathbf{h}_{i}$ are orthonormal and the idempotent projection matrix takes the form $\mathbf{P}_{H}=\mathbf{H}\left(\mathbf{H}^{H} \mathbf{H}\right)^{-1} \mathbf{H}^{H}=\mathbf{H} \mathbf{H}^{H}$ and the matrix projection on the $i$ 'th axis $\left(\mathbf{P}_{H}\right)_{i}=\mathbf{h}_{i} \mathbf{h}_{i}^{H}$. In this paper, we will use the DFT matrix $\mathbf{H}$ as the approximation of the eigenvector's matrix $\mathbf{U}$

$$
\mathbf{H}=\left[\begin{array}{cccc}
1 & 1 & \cdots & 1 \\
z_{0}^{1} & z_{1}^{1} & \cdots & z_{N-1}^{1} \\
\vdots & \vdots & \vdots & \vdots \\
z_{0}^{N-1} & z_{1}^{N-1} & \cdots & z_{N-1}^{N-1}
\end{array}\right],
$$

where $h_{i, n}=z_{i}^{n}=\frac{1}{\sqrt{N}} \exp \left(\frac{j 2 \pi i n}{K}\right), j=\sqrt{-1}$. In this work, we consider the input signals' space $\langle\mathbf{H}\rangle$ that is equal to the background plus noise covariance matrix eigenvector's space.

\subsection{Object Subspace and Model}

The received signal from the small fixed object in successive frames is often modeled using a single zero frequency spectral line. However, since the object is a floating object on the agitated sea surface, the spectral radiance is affected by the object oscillations. Therefore, the spectrum of reflections from a floating object consists of several low-frequency lines. We define the $N \times p$ matrix $\mathbf{H}_{s}=\left[\mathbf{h}_{0}, \mathbf{h}_{1}, \cdots, \mathbf{h}_{p-1}\right]$ and its corresponding object subspace $\left\langle\mathbf{H}_{s}\right\rangle$, which is the span of $\left\{\mathbf{h}_{i}\right\}_{0}^{p-1}$. We next define the $N \times(N-p)$ matrix, $\mathbf{H}_{s}^{\perp}=\left[\mathbf{h}_{p}, \mathbf{h}_{p+1}, \cdots, \mathbf{h}_{N-1}\right]$, and its corresponding subspace $\left\langle\mathbf{H}_{s}^{\perp}\right\rangle$, which is the span of $\left\{\mathbf{h}_{i}\right\}_{p}^{N-1}$. We assume that $\mathbf{H}_{s}$ and $\mathbf{H}_{s}^{\perp}$ are, respectively, full rank and that $\mathbf{H}_{s}^{\perp H} \mathbf{H}_{s}=0$. Let $\mathbf{H}_{s}$ be the $N \times p$ mode matrix with columns that contain the orthogonal basis vectors that span the object subspace, $p<N$. The object signal $\mathbf{s}=$ $\mathbf{H}_{s} \boldsymbol{\theta}=\left[\begin{array}{llll}s_{1} & s_{2} & \cdots & s_{N}\end{array}\right]^{T}$ is the deterministic unknown signal of interest, which belongs to a known subspace of the eigenvectors $\left\langle\mathbf{H}_{s}\right\rangle$ of size $N \times p$, where the abundance vector $\boldsymbol{\theta}$ (size $p \times 1)$ is unknown. The orthogonal subspace $\left\langle\mathbf{H}_{s}^{\perp}\right\rangle$ contains the columns from $p$ to $N-1$. The projection matrix onto subspace $\left\langle\mathbf{H}_{s}\right\rangle$ is equal to $\mathbf{P}_{s}=\mathbf{H}_{s} \mathbf{H}_{s}^{H}$ and onto orthogonal subspace $\left\langle\mathbf{H}_{s}^{\perp}\right\rangle$ is equal to $\mathbf{P}_{s}^{\perp}=\mathbf{H}_{s}^{\perp}\left(\mathbf{H}_{s}^{\perp}\right)^{H}$.

\subsection{Subpixel Target Detection Problem}

The problem is to find the best algorithm for detecting a partially known relatively small target signature in a given image sequence. We consider $N$ sequential images of the analyzed video. We select one pixel with the same coordinates on each image. We are processing the vector of the received signal $\mathbf{x}=\left[\begin{array}{llll}x_{1} & x_{2} & \cdots & x_{N}\end{array}\right]^{T}$. We assume that an analyzed pixel is partially filled by the object, that is, we focus attention on a subpixel or nonresolved small objects. We develop a hypothesis test that distinguishes the signal-plus-background-plus-noise statistical hypothesis $\left(H_{1}\right)$ from the background-plus-noise statistical hypothesis $\left(H_{0}\right)$. We can represent two statistical hypotheses

$$
\left\{\begin{array}{l}
H_{0}: \mathbf{x}=\mathbf{c}+\mathbf{n} \\
H_{1}: \mathbf{x}=b \mathbf{s}+(1-b) \mathbf{c}+\mathbf{n}
\end{array}\right.
$$

where $\mathbf{c}=\left[\begin{array}{llll}c_{1} & c_{2} & \cdots & c_{N}\end{array}\right]^{T}$ is the correlated Gaussian background clutter vector $\mathbf{c} \sim N\left(\mathbf{m}, \mathbf{R}_{b}\right)$, $\mathbf{n}=\left[\begin{array}{llll}n_{1} n_{2} & \cdots & n_{N}\end{array}\right]^{T}$ is the uncorrelated Gaussian channel noise vector $\mathbf{n} \sim N\left(0, \sigma_{n}^{2} \mathbf{I}\right)$, $\mathbf{s}=\left[\begin{array}{llll}s_{1} s_{2} & \cdots & s_{N}\end{array}\right]^{T}$ is the object unknown deterministic signal vector, and $b \leq 1$ is the object fill factor (the relation between the pixel area occupied by an object and total pixel area). Under $H_{0}$ i.e., the background-plus-noise hypothesis, we have $\mathbf{x} \sim N\left(\mathbf{m}, \mathbf{R}_{b n}\right)$, where $\mathbf{R}_{b n}=\mathbf{R}_{b}+\sigma_{n}^{2} \mathbf{I}$, and under $H_{1}$, i.e., the signal-plus-background-and-noise hypothesis 
$\mathbf{x} \sim N\left[b \mathbf{s}+(1-b) \mathbf{m}, \mathbf{R}_{s b n}\right]$, where covariance matrix $\mathbf{R}_{s b n}=(1-b)^{2} \mathbf{R}_{b}+\sigma_{n}^{2} \mathbf{I}$ and a priori unknown. Note that we assume the homogeneous Gaussian environment and assume that the secondary data set is available to estimate the background-plus-noise mean $\mathbf{m}$ and covariance matrix $\mathbf{R}_{b n}$ under $H_{0}$. The covariance matrix $\mathbf{R}_{s b n}$ of the received signal under hypothesis $H_{1}$ depends on object fill factor $b$, background covariance matrix $\mathbf{R}_{b}$, and channel noise variance $\sigma_{n}^{2}$. In the case of $b=1$, the background covariance matrix of the received signal under $H_{1}$ is equal to the uncorrelated Gaussian noise case: $\mathbf{R}_{s b n}=\sigma_{n}^{2} \mathbf{I}$. We assume that the primary data set does not allow to estimate the $\mathbf{R}_{s b n}$ under $H_{1}$ completely, but it is possible to estimate PSD for the frequencies $\left(f_{i} \geq p / N\right)$ that do not contain reflection components from the object. It is well known that the reflections from a floating object contain only low-frequency spectral components in comparison with reflections from an agitated sea surface. In this paper, we will use this case for GLRT synthesis.

\subsection{GLR Tests in the Case of Known and Unknown Background Power; Normalized Covariance Matrices $\boldsymbol{R}_{b n}$ and $\boldsymbol{R}_{s b n}$ Are Known}

The GLRT synthesis requires calculating of maximum likelihood estimate (MLE) unknown parameters, then estimating and substituting them in the likelihood equation. We cannot use the well-known whitening method because the background covariance matrices under $H_{0}$ and $H_{1}$ are different. In this section, we consider the case where the MLE of the background spectrum using primary data is impossible, for example, when the spectral range of the reflections from the background and the object coincide. We also assume that object fill factor $b=1$ and normalized covariance matrices $\mathbf{R}_{s b n}, \mathbf{R}_{b n}$, and background power are $a$ priori known. The GLR statistic for the problem in hand is

$$
L=\frac{\max _{\boldsymbol{\theta}} p_{1}(\mathbf{x})}{p_{0}(\mathbf{x})}
$$

where $p_{1}(\mathbf{x})$ and $p_{0}(\mathbf{x})$ are the probability density functions (pdfs) under hypotheses $H_{1}$ and $H_{0}$, respectively. It is well known ${ }^{14}$ that the log likelihood ratio is then

$$
\ln (L)=\ln \frac{\left(\operatorname{det} \mathbf{R}_{b n}\right)^{1 / 2}}{\left(\operatorname{det} \mathbf{R}_{s b n}\right)^{1 / 2}}-\frac{1}{2}\left[\left(\mathbf{x}-\mathbf{m}-\mathbf{H}_{s} \hat{\boldsymbol{\theta}}\right)^{T} \boldsymbol{R}_{s b n}^{-1}\left(\mathbf{x}-\mathbf{m}-\mathbf{H}_{s} \hat{\boldsymbol{\theta}}\right)-(\mathbf{x}-\mathbf{m})^{T} \mathbf{R}_{b n}^{-1}(\mathbf{x}-\mathbf{m})\right],
$$

where MLE of the abundance vector

$$
\hat{\boldsymbol{\theta}}=\mathbf{H}_{s}^{H}(\mathbf{x}-\mathbf{m}),
$$

we will substitute in Eq. (4).

The first term is independent of the data, so we may ignore it and use only the quadratic form

$$
\ln \left(L_{1}\right)=(\mathbf{x}-\mathbf{m})^{T} \mathbf{R}_{b n}^{-1}(\mathbf{x}-\mathbf{m})-(\mathbf{x}-\mathbf{m})^{T} \mathbf{P}_{s}^{\perp} \mathbf{R}_{s b n}^{-1} \mathbf{P}_{s}^{\perp}(\mathbf{x}-\mathbf{m}),
$$

where $\mathbf{P}_{s}^{\perp}=\mathbf{I}-\mathbf{P}_{s}$ projection matrix on the subspace orthogonal to subspace $\left\langle\mathbf{H}_{\mathbf{s}}\right\rangle$. The first and second terms in Eq. (6) can be represented in following forms:

$$
\begin{gathered}
(\mathbf{x}-\mathbf{m})^{T} \mathbf{R}_{b n}^{-1}(\mathbf{x}-\mathbf{m})=(\mathbf{x}-\mathbf{m})^{T} \mathbf{H} \mathbf{H}^{H} \mathbf{R}_{b n}^{-1} \mathbf{H} \mathbf{H}^{H}(\mathbf{x}-\mathbf{m}), \\
(\mathbf{x}-\mathbf{m})^{T} \mathbf{P}_{s}^{\perp} \mathbf{R}_{s b n}^{-1} \mathbf{P}_{s}^{\perp}(\mathbf{x}-\mathbf{m})=(\mathbf{x}-\mathbf{m})^{T} \mathbf{H}_{s}^{\perp}\left(\mathbf{H}_{s}^{\perp}\right)^{H} \mathbf{R}_{s b n}^{-1} \mathbf{H}_{s}^{\perp}\left(\mathbf{H}_{s}^{\perp}\right)^{H}(\mathbf{x}-\mathbf{m}) .
\end{gathered}
$$

Using the spectral representation of the signals and covariance matrices, we may simplify the quadratic form [Eq. (6)]. We use Hotelling transform and obtain the complex uncorrelated vector $\mathbf{y}$ 


$$
\mathbf{y}=\mathbf{H}^{H}(\mathbf{x}-\mathbf{m})=\left[\begin{array}{llll}
y_{0} & y_{1} & \ldots & y_{N-1}
\end{array}\right]^{T} .
$$

The inverse covariance matrices in Eqs. (7) and (8) take a diagonal form

$$
\begin{gathered}
\mathbf{H}^{H} \mathbf{R}_{b n}^{-1} \mathbf{H}=\mathbf{D}_{b n}^{-1}=\operatorname{diag}\left[\lambda_{b n 0}^{-2}, \lambda_{b n 1}^{-2}, \ldots, \lambda_{b n(N-1)}^{-2}\right], \\
\left(\mathbf{H}_{s}^{\perp}\right)^{H} \mathbf{R}_{s b n}^{-1} \mathbf{H}_{s}^{\perp}=\mathbf{D}_{s b n}^{-1}=\operatorname{diag}\left[0, \ldots, 0, \lambda_{s b n(p)}^{-2}, \ldots, \lambda_{s b n(N-1)}^{-2}\right],
\end{gathered}
$$

where $\lambda_{b n i}^{2}=P_{b n}\left(f_{i}\right)$ are the eigenvalues (PSD) of the $\mathbf{R}_{b n}$, and $\lambda_{s b n i}^{2}=P_{s b n}\left(f_{i}\right)$ are the eigenvalues (PSD) of the $\mathbf{R}_{s b n}$. The second term in Eq. (6) can be simplified

$$
(\mathbf{x}-\mathbf{m})^{T} \mathbf{P}_{s}^{\perp} \mathbf{R}_{s b n}^{-1} \mathbf{P}_{s}^{\perp}(\mathbf{x}-\mathbf{m})=\sum_{i=p}^{N-1} \frac{y_{i} y_{i}^{*}}{\lambda_{s b n i}^{2}} .
$$

Then, we obtain the following statistic test:

$$
\ln \left(L_{1}\right)=\sum_{i=0}^{N-1} \frac{y_{i} y_{i}^{*}}{\lambda_{b n i}^{2}}-\sum_{i=p}^{N-1} \frac{y_{i} y_{i}^{*}}{\lambda_{s b n i}^{2}}{ }^{>}{ }_{H_{0}}^{H_{1}} \eta,
$$

where ()$^{*}$ is the complex conjugate value and $\eta$ is the threshold. This GLRT is known as the spectrum matching detector ${ }^{16}$ (SpMD).

The GLR statistic in the case of unknown background power $\sigma_{1}^{2}$ under $H_{1}$ is

$$
L_{u n}=\frac{\max _{\boldsymbol{\theta}, \sigma_{1}^{2}} p_{1}\left(\mathbf{x}, \theta, \sigma_{1}^{2}\right)}{p_{0}(\mathbf{x})} .
$$

The GLRT-based detector named HMSD was obtained in Ref. 27 in the following form:

$$
D_{\mathrm{HMSD}}(\mathbf{y})=\frac{m\left\|\mathbf{y}_{t}\right\|^{2}}{N \sigma_{0}^{2}}-\ln \frac{\left\|\mathbf{y}_{t}^{\perp}\right\|^{2}}{(N-p) \sigma_{0}^{2}},
$$

where $m$ is the sensitive factor, $\mathbf{y}_{t}=\mathbf{P}_{s} \mathbf{y}, \mathbf{y}_{t}^{\perp}=\mathbf{P}_{s}^{\perp} \mathbf{y}$.

\subsection{GLRT in the Case of Unknown Covariance Matrix $\boldsymbol{R}_{s b n}$}

It is well known that GLRTs are obtained by inserting MLE for unknown parameters into the likelihood ratio. In the case of some unknown parameters of the pdf, it must be estimated under the alternative hypothesis, i.e., in our case the detector should be able to estimate the $\mathbf{R}_{s b n}$ using the received primary signal $\mathbf{x}$. But the received signal $\mathbf{x}$ under the alternative hypothesis contains the sum of the useful signal from the object-plus channel noise and the background clutter $(b<1)$. The presence of the useful signal $b \mathbf{s}$ with unknown parameters in the received sample does not make it possible to estimate the $\mathbf{R}_{s b n}$. In this paper, we use the physically meaningful phenomenon where the maximum spectrum frequency $f_{i}=(p-1) / N$ of the received signal from the floating object is less than the maximum frequency from the sea surface $f_{i}=(N-1) / N$.

In practice, the frame rate in the camera should be sufficient to obtain a spectral range in which it is possible to separate the first low-frequency region $\left[0 \leq f_{i}<(p / N)\right]$, where there is energy from the object and the background plus noise, and the second frequency region $\left[\frac{p}{N} \leq f_{i} \leq(N-1) / N\right]$, where there is no energy from the object. Since the MLE of the covariance matrix $\mathbf{R}_{s b n}$ is not completely available in these conditions using the primary data set, we can replace the MLE of the covariance matrix by the MLE of the eigenvalues of the covariance matrix. Under these conditions, the MLEs of the eigenvalues are available through estimates of the received signal PSD $P_{x}\left(f_{i}\right)$, where $i \in(p, N-1)$.

It is well known that GLRTs are obtained by inserting MLE for unknown parameters into the likelihood ratio. The GLRT is obtained by considering the joint pdf of the measurement $\mathbf{x}$ under $H_{0}$ and $H_{1}$. The GLRT expression for the problem in hand is 


$$
L_{2}=\frac{\max _{\lambda_{s b n i}^{2}, \boldsymbol{\theta}} p_{1}\left(\mathbf{x} \mid H_{1}\right)}{p_{0}\left(\mathbf{x} \mid H_{0}\right)}>_{H_{0}}^{H_{1}} \eta_{2}
$$

where $\eta_{2}$ is the threshold. The determinant of the background-plus-noise covariance matrix $\operatorname{det}\left(\mathbf{R}_{b n}\right)=\prod_{i=0}^{N-1} \lambda_{b n i}^{2}$ is available using the secondary data set and for the signal-plusbackground-plus noise covariance matrix $\operatorname{det}\left(\mathbf{R}_{s b n}\right)=\prod_{i=0}^{N-1} \lambda_{s b n i}^{2}$. Under hypothesis $\mathbf{H}_{1}$, it is possible to obtain the MLE of the $\hat{\lambda}_{s b n i}^{2}$ only for $i=p, \ldots, N-1$

$$
\hat{\lambda}_{s b n i}^{2}=y_{i} y_{i}^{*}
$$

The log likelihood ratio is approximately

$$
\ln \left(L_{2}\right)=\ln \frac{\prod_{i=0}^{N-1} \lambda_{b n i}^{2}}{\prod_{i=p}^{N-1} y_{i} y_{i}^{*}}+\sum_{i=0}^{N-1} \frac{y_{i} y_{i}^{*}}{\lambda_{b n i}^{2}}-(N-p) .
$$

We can obtain MSpMD

$$
\sum_{i=0}^{N-1} \frac{y_{i} y_{i}^{*}}{\lambda_{b n i}^{2}}-\sum_{i=p}^{N-1} \ln \left(\frac{y_{i} y_{i}^{*}}{\lambda_{b n i}^{2}}\right){ }_{{ }_{H_{0}}}^{{ }^{H_{1}}} \eta_{3},
$$

where $\eta_{3}$ is the threshold. We can see from Eq. (19) that the detection quality depends on the relation between the target spectra contribution (the first term) and the background spectra change contribution (the second term). Note that the detector SpMD [Eq. (13)] uses only changes in target energy, and the detector 2 uses changes in target energy and changes in background energy but does not use change in the shape of the background spectrum under hypothesis $H_{1}$.

\section{Performance Assessment}

To test the effectiveness of the MSpMD, a series of experiments is conducted on the synthetic and real data sets. The performance of MSpMD is compared with the well-known SpMD and the recently proposed HMSD. The experimental platform is a PC with Intel Core i5, $2.20 \mathrm{GHz}, 4 \mathrm{G}$ memory, and 64-bit Windows 10. All algorithms are implemented by MATLAB ${ }^{\circledR} 2015 \mathrm{a}$. Since closed-form expressions for the false alarm probability $P_{F}$ and the detection probability $P_{D}$ are not available for the MSpMD, we evaluated them resorting to standard Monte Carlo techniques based on $100 / P_{F}$ and $100 / P_{D}$ independent trials, respectively. In order to limit the computational burden, we set $P_{F}=10^{-3}$. We assess the performances of the MSpMD [Eq. (19)], the well-known SpMD ${ }^{15}$ [Eq. (13)], and the recently proposed HMSD [Eq. (15)] in the case of the difference between statistical parameters (the means, the variances, and covariances) of the unstructured background and objects. At the analysis stage, one must specify the background-plus-noise correlation properties as well as the other statistical properties of the background and the object. As to the background normalized covariance matrix, we assume that it has an exponential shape, i.e., $\mathbf{R}_{b}=\left[r_{i, k}\right]=\left[\rho^{|i-k|}\right]$, where $\rho=(0.5-0.9)$ is the one-lag correlation coefficient. As to the useful primary data, it is now modeled as the deterministic target signal with $p$ independent basis modes (the target modes matrix $\mathbf{H}$ is a Vandermonde matrix with discrete complex exponential elements) and the abundance vector $\boldsymbol{\theta}$. The parameter $\boldsymbol{\theta}$ is unknown in practice, but for our scenario, it is possible to use the appropriate deterministic approximation $\boldsymbol{\theta} \in(0,1)$.

The detection performance of the analyzed detectors depends on several parameters: (1) the difference between the statistical means of reflections from the sea and from the target, (2) the ratio of the variances of the signals from the sea and from the target, (3) the ratio of channel noise variances and reflections from the sea surface, (4) correlation factor of the reflections from the sea surface, (5) object fill factor, (6) sample size $N$, and (7) estimated number $p$ of harmonics in a signal reflected from a floating object. It is obvious that in the absence of differences in the 


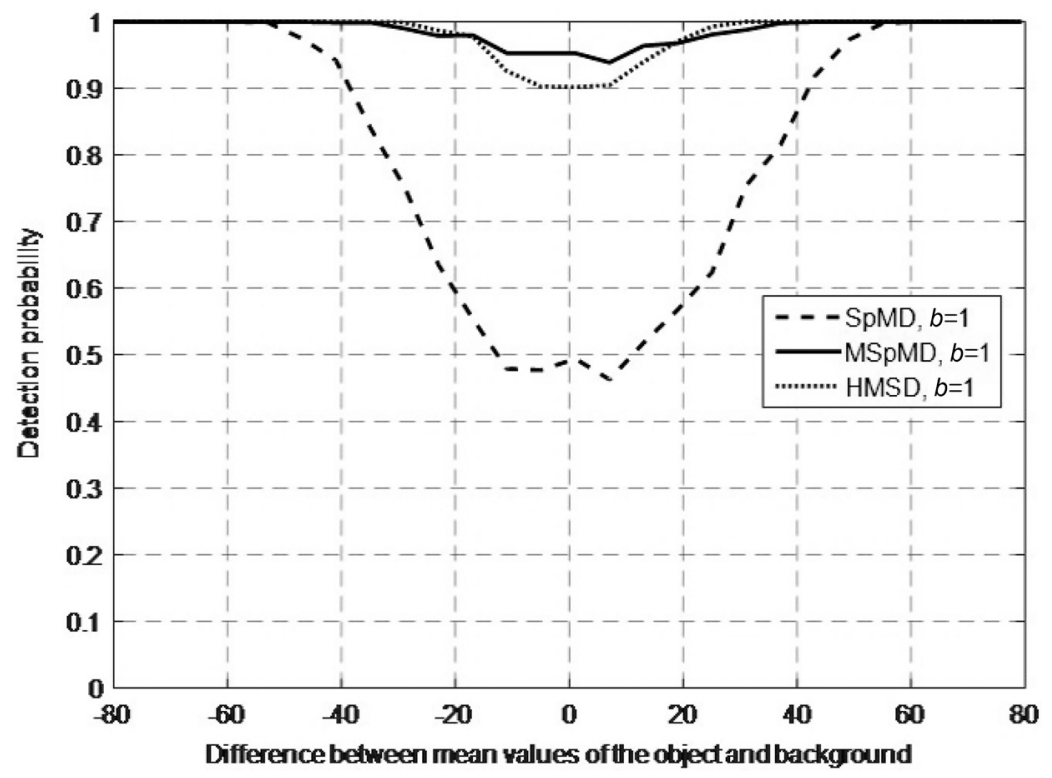

Fig. 1 Detection probability versus difference between mean values of the object and background for the object fill factor $b=1$, false alarm probability $F=0.001$, channel variance/background variance $=0.005$, object variance/background variance $=1, p=3, N=10, \rho=0.85$ (simulated data set).

means and variances of the object and the background, the object (Gauss model) can be detected only in the presence of the differences in the covariance matrix or in the spectral composition.

In Fig. 1, the detection probability $P_{D}$ is shown as a function of the difference between mean values (measured in entire units between -255 and 255) of the reflections from the object and background for object fill factors $b=1$ for three detectors: SpMD, MSpMD, and HMSD. The ratio between an object variance and background variance is equal to 1 . These curves show the fact that MSpMD has a detection probability more than 0.93 even when there is no difference in the average values and variances of the object and background. This is because the MSpMD is sensitive to differences between a maximum frequency of the object and background reflections. The second term in Eq. (19) depends on the ratio of the energy spectra of the received signals at frequencies higher than the maximum object frequency in the presence and absence of an object. The received signals reflected from the sea plus channel noise are present under the null hypothesis, and in the presence of an object (alternative hypothesis), only channel noise is present $(b=1)$ on frequencies higher than the maximum object frequency. The SpMD is sensitive only to the contrast of the means and variances of the object and background and hence SpMD cannot detect the object in these conditions. The HMSD is also sensitive to contrast between the background and channel noise variances under null and alternative hypotheses at high frequencies. But the HMSD is not sensitive to changes in the shape of the background spectrum on frequencies higher than the maximum object frequency. Therefore, the detection efficiency of the HMSD is inferior to that of proposed detector MSpMD, which is sensitive to the mean, variance, and different spectrum shape of the received signal under null and alternative hypotheses. In Fig. 2, the object fill factor $b=0.8$ with respect to $b=1$ in the case of Fig. 1 . All other object and background parameters are the same. One can see that the increasing the object fill factor for detectors increases the quality of detection for both detectors. Analysis of the graphs in Fig. 3 shows that increasing the sample size $N$ significantly increases the detection probability of the MSpMD. In Figs. 4 and 5, the performance of the MSpMD at object fill factor $b=1$ and 0.6 is significantly higher than HMSFD and SpMD performances, respectively. Figures 4 and 6 show that the detection probability depends on the ratio between channel and background variances (0.01 and 0.04, respectively). This comparison shows that channel noise significantly affects the quality of the all detectors, but only the MSpMD's performance remains high. In Fig. 7, the detection probability is shown as a function of the difference between mean values of the 


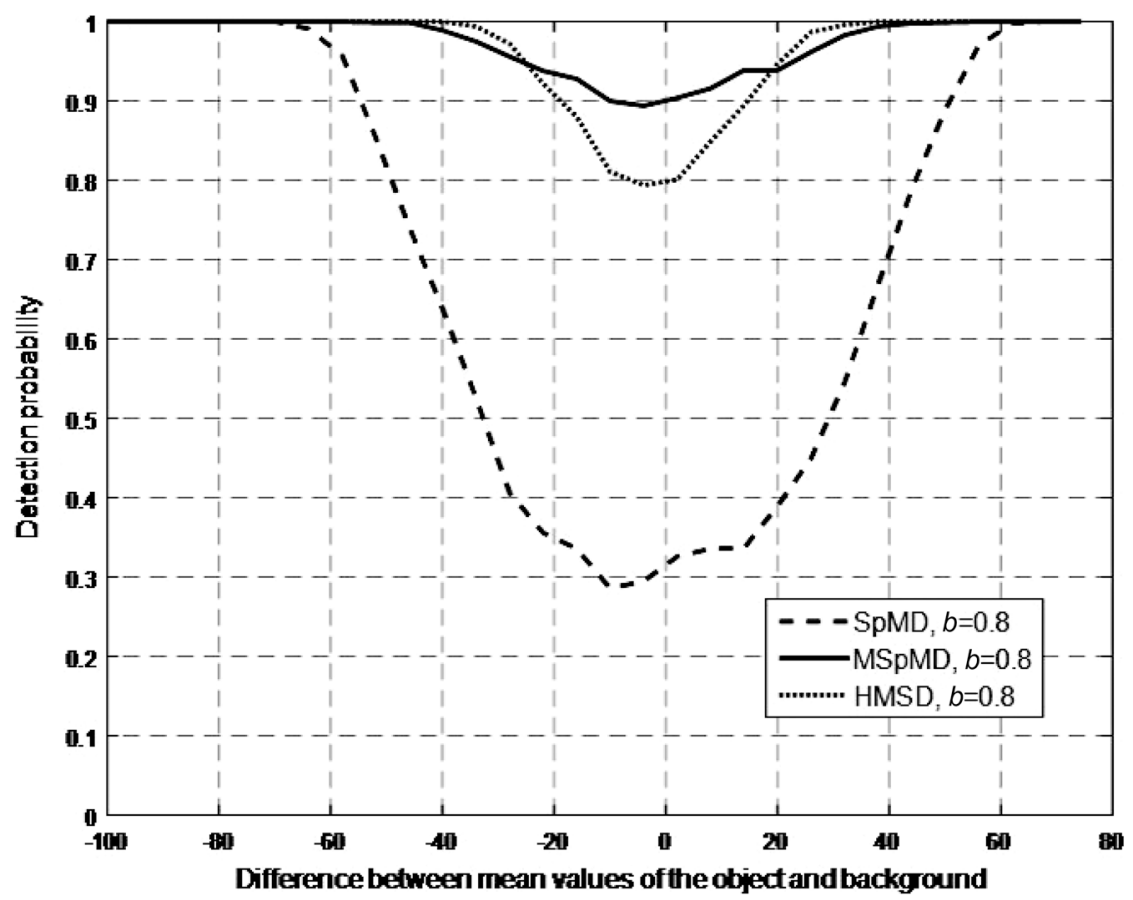

Fig. 2 Detection probability versus difference between mean values of the object and background for the object fill factor $b=0.8$, false alarm probability $F=0.001$, channel variance/background variance $=0.005$, object variance/background variance $=1, p=3, N=10, \rho=0.85$ (simulated data set).

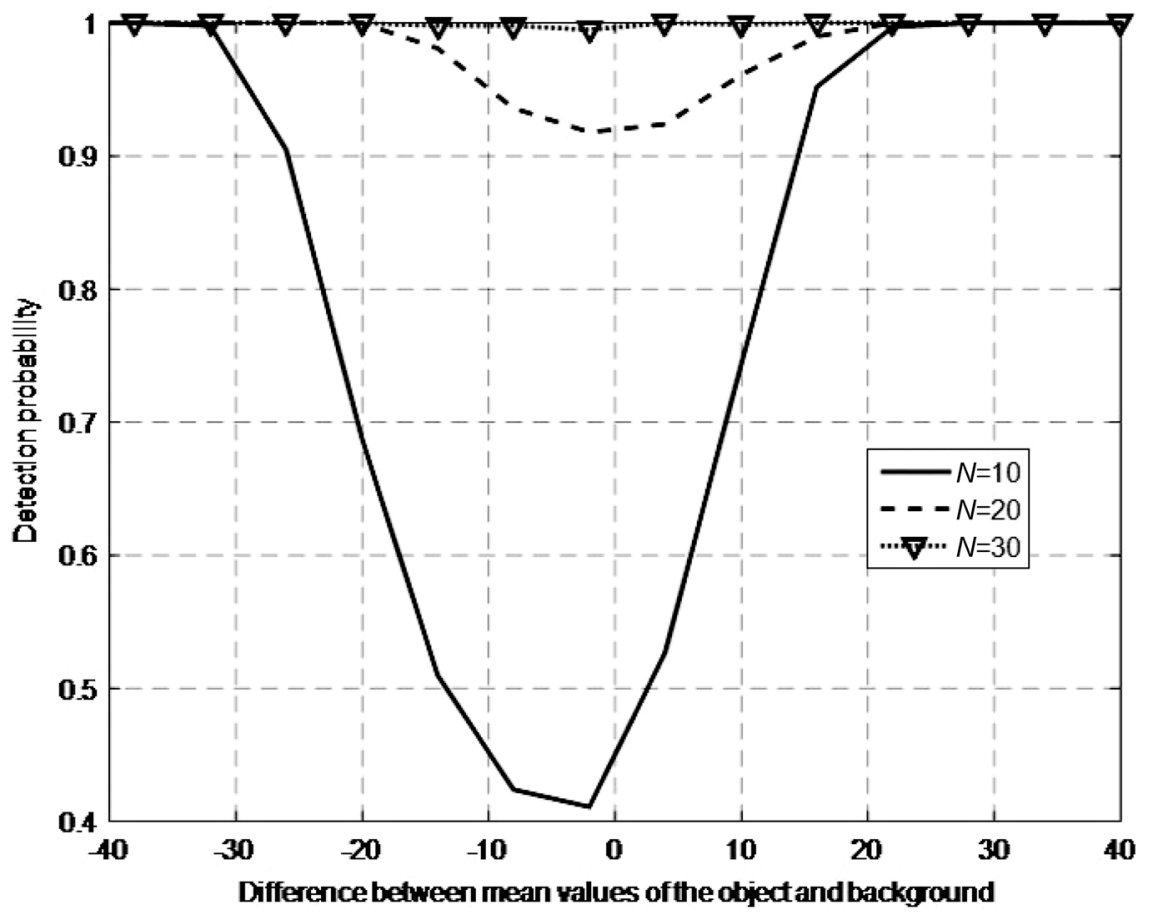

Fig. $3 \mathrm{MSpMD}$ detection probability versus difference between mean values of the object and background for different sample sizes $N=10,20,30, b=1, F=0.001$, channel variance/background variance $=0.005$, object variance/background variance $=1, p=5, N=20, \rho=0.85$ (simulated data set). 


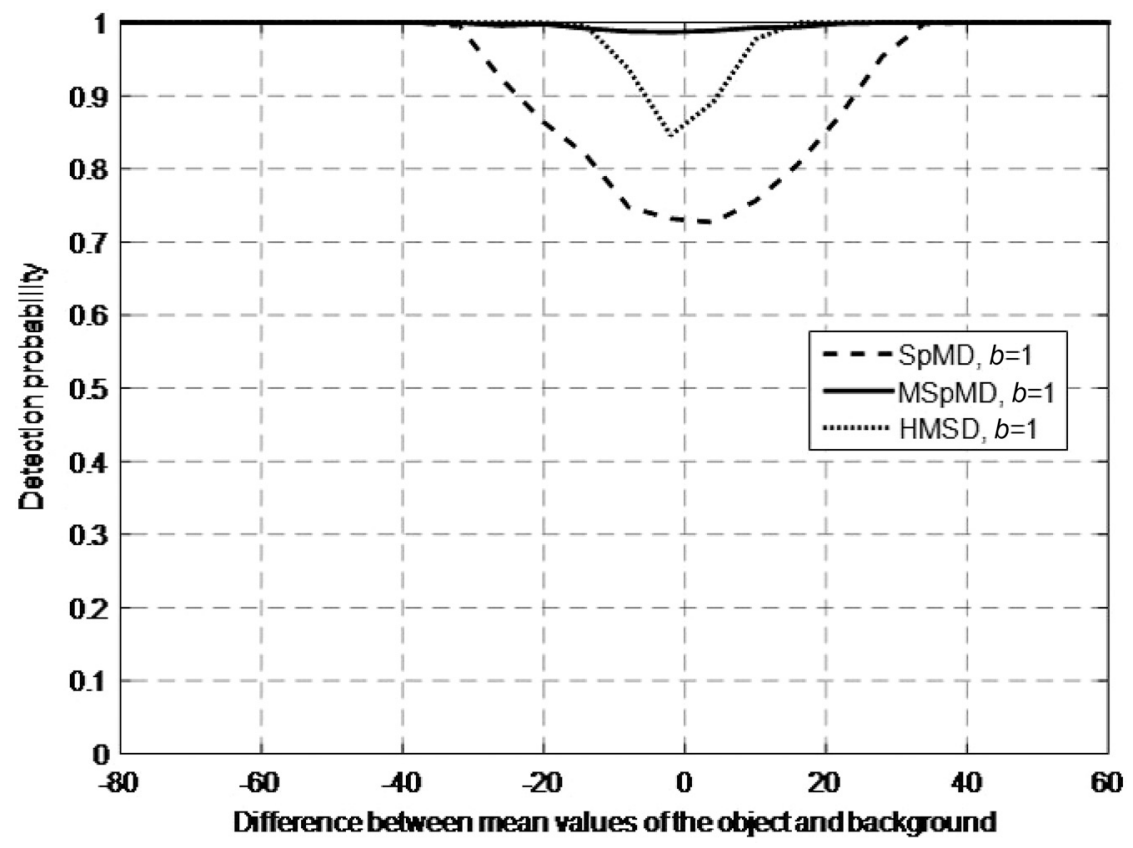

Fig. 4 Detection probability versus difference between mean values of the object and background for object fill factor $b=1, F=0.001$, channel variance/background variance $=0.01$, object variance/background variance $=1, p=5, N=20, \rho=0.85$ (simulated data set).

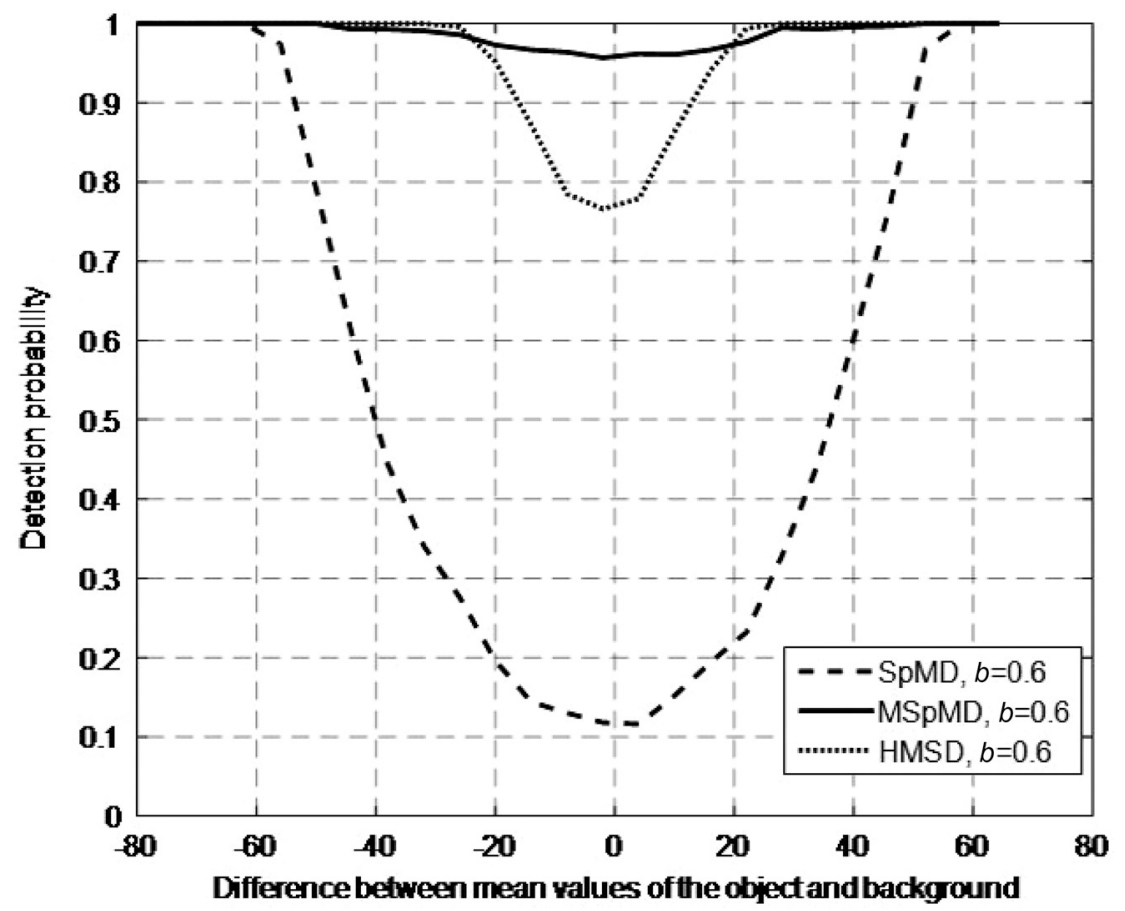

Fig. 5 Detection probability versus difference between mean values of the object and background for object fill factor $b=0.6, F=0.001$, channel variance/background variance $=0.01$, object variance/background variance $=1, p=5, N=20, \rho=0.85$ (simulated data set).

reflections from the object and background for object fill factors $b=1$ at changing of the maximum frequency $p$ of the reflection from the object. When the maximum frequency $p$ is increased, the first term [Eq. (19)] is increased, but the second term [Eq. (19)] is decreased. Consequently, the detection probability can have the maximum when the valor $p$ is between 1 and maximum. In Fig. 8, the detection probability versus false alarm probability curves 


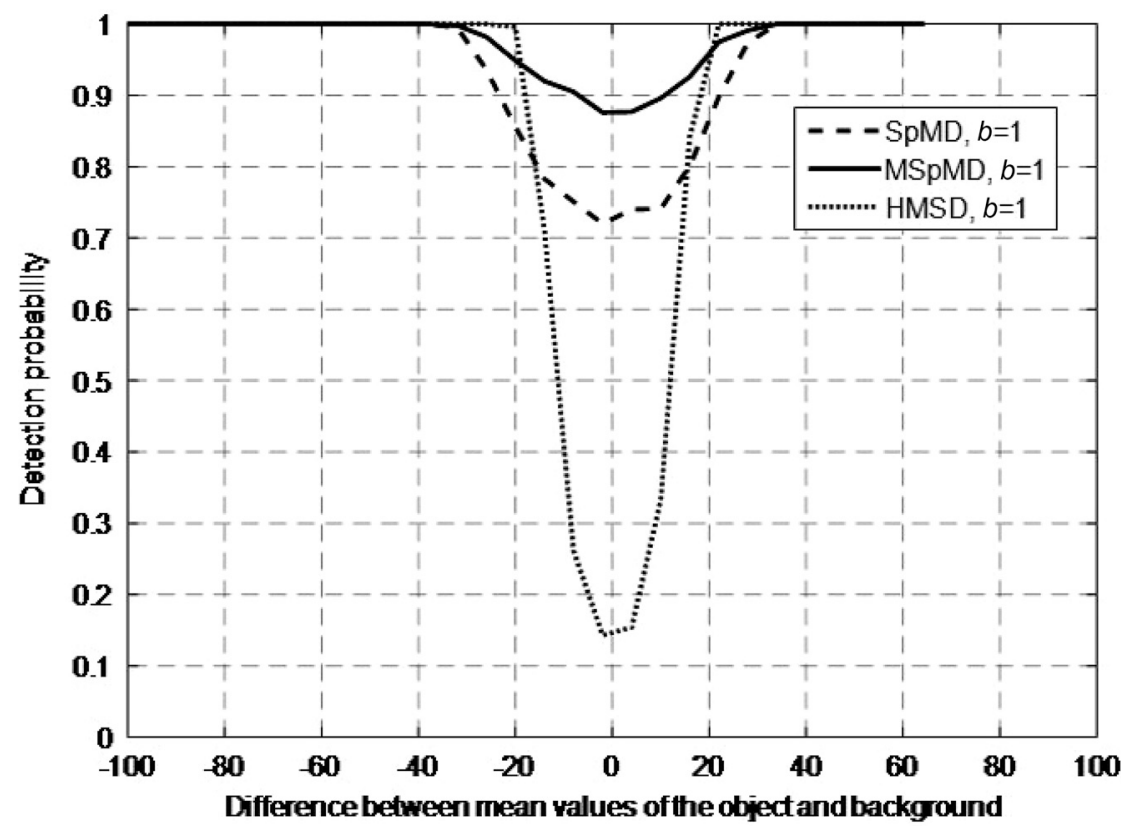

Fig. 6 Detection probability versus difference between mean values of the object and background for object fill factor $b=1, F=0.001$, channel variance/background variance $=0.04$, object variance/background variance $=1, p=5, N=20, \rho=0.85$ (simulated data set).

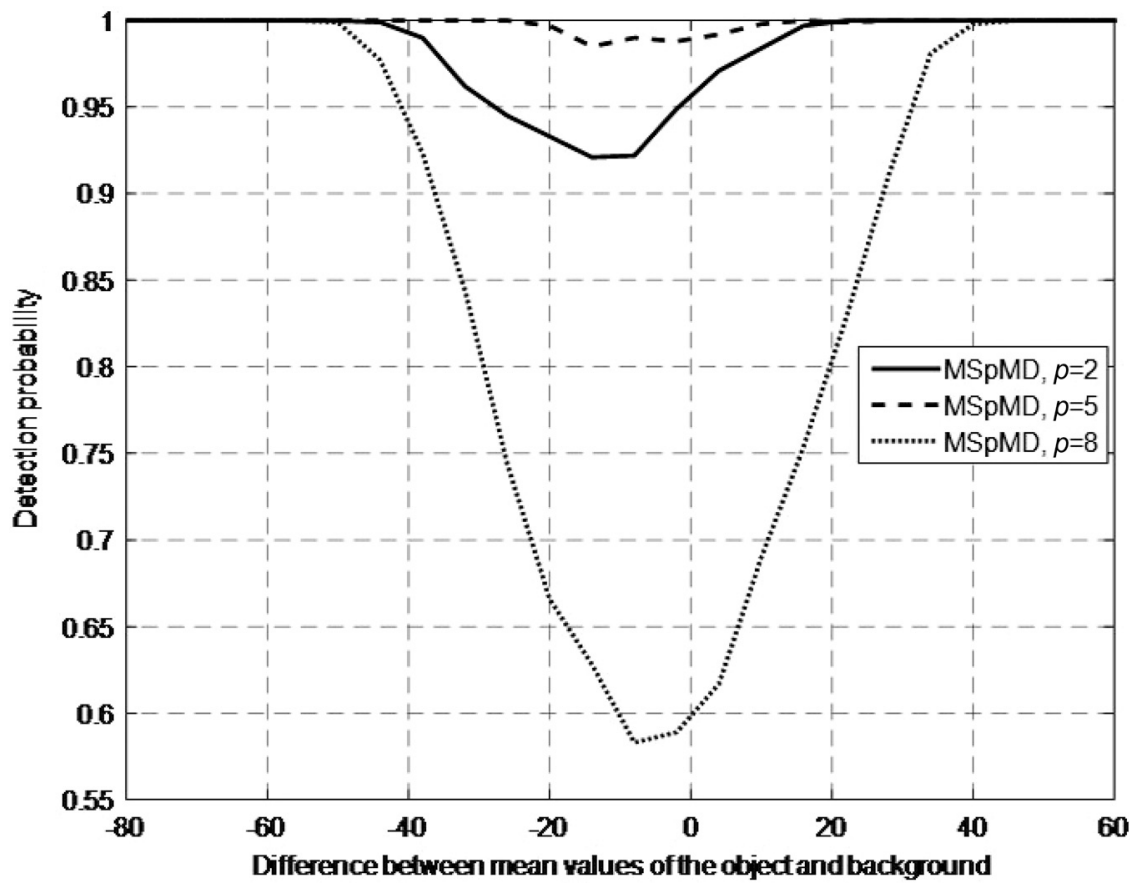

Fig. 7 MSpMD detection probability versus difference between mean values of the object and background for different number $p$ of the object harmonics, $b=1, F=0.001$, channel variance/background variance $=0.02$, object variance/background variance $=1, \rho=0.8, N=20$ (simulated data set). 


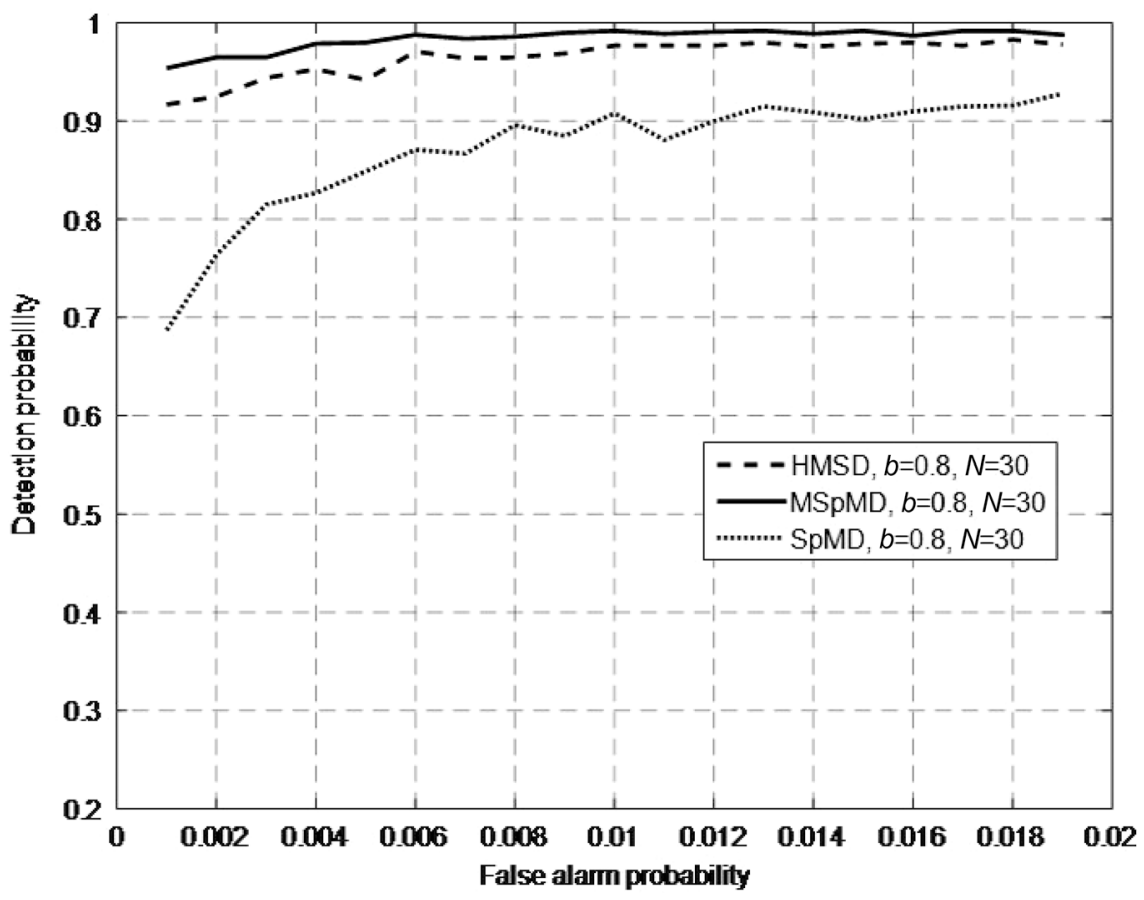

Fig. 8 Detection probability versus false alarm probability for different $N, b=0.8, p=8$, channel variance/background variance $=0.01$, object variance/background variance $=1, \rho=0.9, N=30$ (simulated data set).

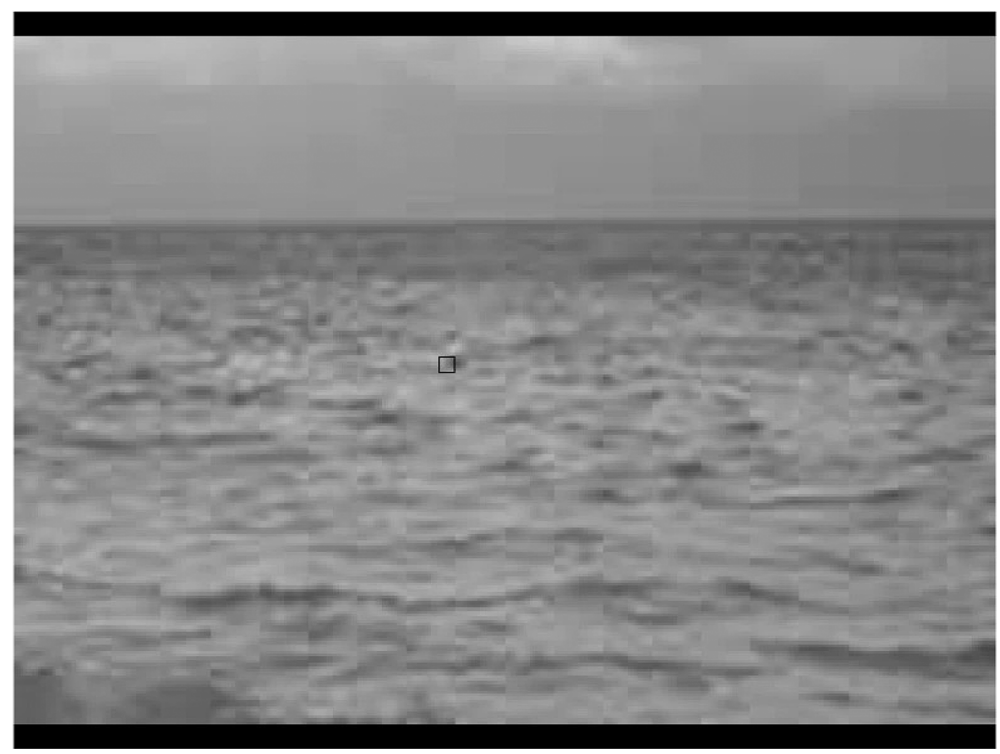

Fig. 9 An image of a floating plastic container 1 (marked observed region is $5 \times 5$ pixels).

(ROC, receiver operating characteristic) show that the detection probability is decreased at small values of the false alarm probability, and the MSpMD provides a higher detection quality than the HMSD and SpMD.

To test the effectiveness of the MSpMD, HMSD, and SpMD, two series of experiments are conducted on the real dataset. We use two image sequences with 10,000 frames (Figs. 9, 10,13, and 14) using the observed regions: $5 \times 5$ pixels (Fig. 10) and $7 \times 10$ (Fig. 14). The video camera had a velocity equal to 30 frame/s. Figure 11 shows the ROC curves of the floating plastic 


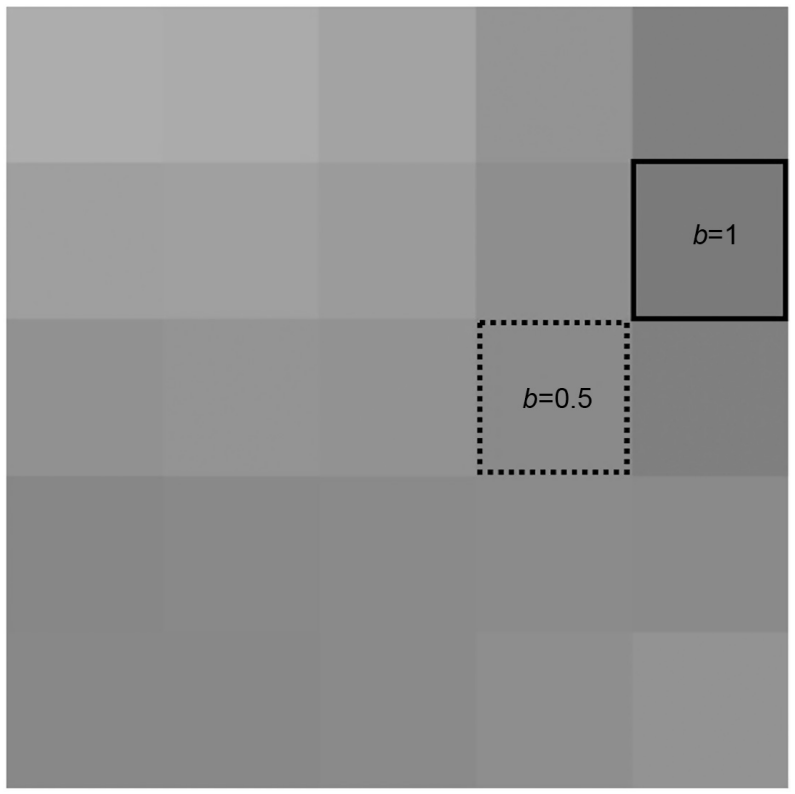

Fig. 10 The observed square region (marked in Fig. 9) with size $5 \times 5$ pixels and two marked pixels with different object fill factors $b$.

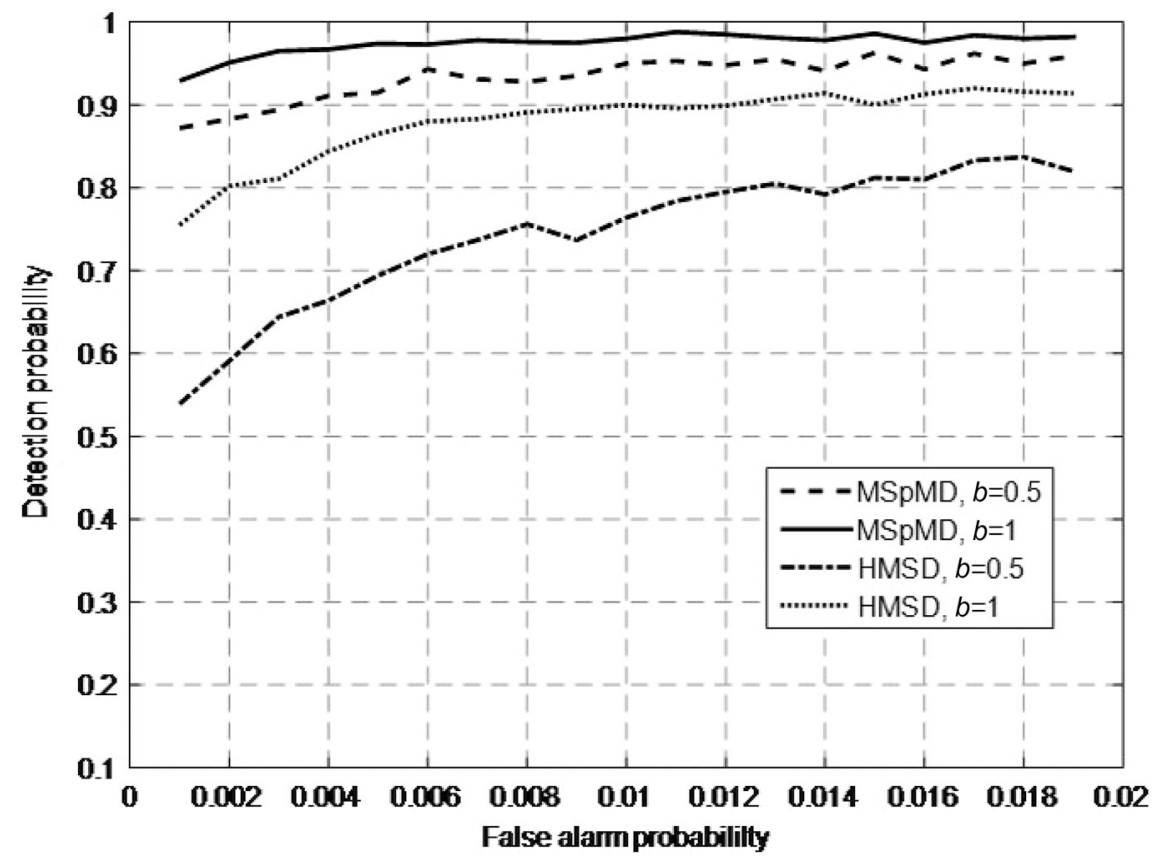

Fig. 11 Real data set (see Figs. 9 and 10). Detection probability versus false alarm probability for different object fill factors for MSpMD and HMSD at $b=1$ and $0.5, N=30, p=5$.

container 1 for the observed region of $5 \times 5$ pixels (see Fig. 10). In this case, the object fill factor is different for different pixels. We consider two pixels' sequences ( $N=30$ frames) marked in Fig. 10 (object fill factor $b=0.5$ and $b=1$ ). The experimental results show that the MSpMD detection performance significantly outperforms the HMSD at any object fill factor. Figures 9 and 10 show the case of the small barely discernible object. Figure 12 shows the ROC curves of the floating plastic container 2 for observed regions of $10 \times 10$ pixels (see Fig. 13). In this case, the object fill factor is different for different pixels. In this case, we consider two pixels' sequences $(N=30$ frames) marked in Fig. 14 (object fill factor $b=0.6$ for pixel 1 and $b=1$ for 


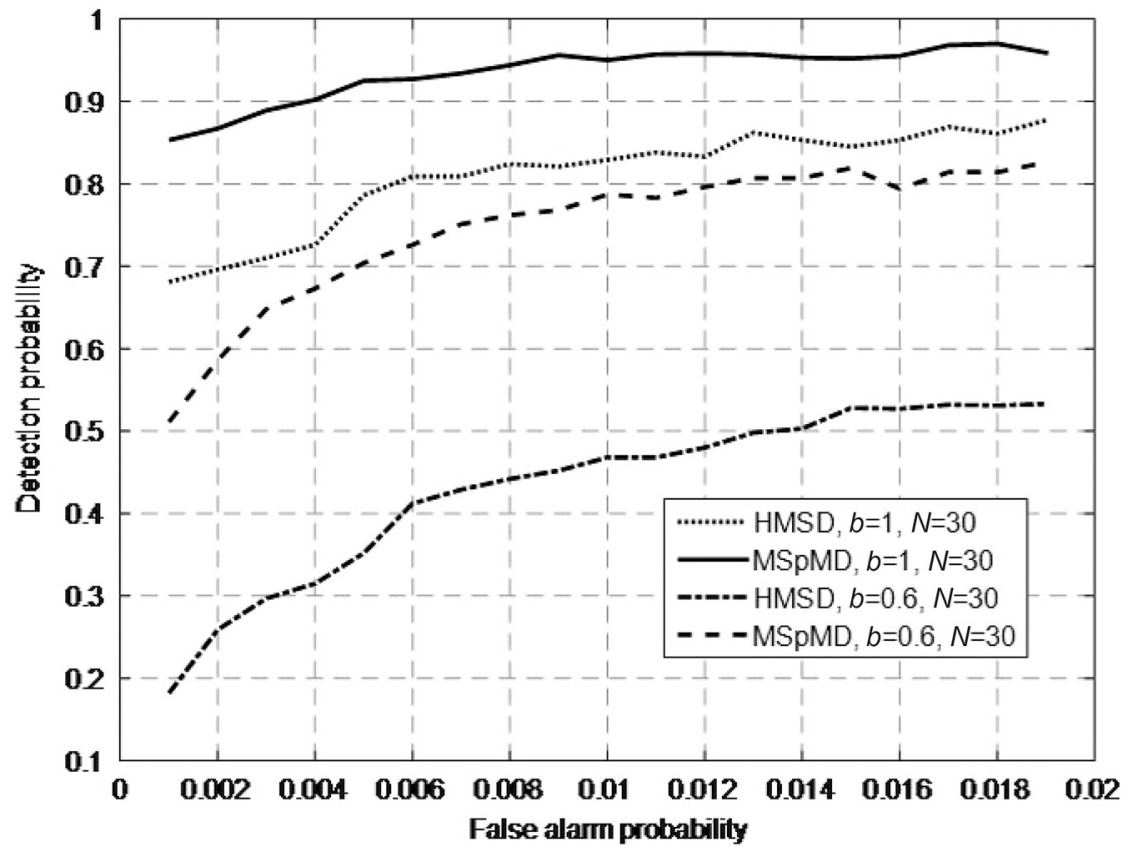

Fig. 12 Real data set (see Figs. 13 and 14). Detection probability versus false alarm probability for MSpMD and HMSD at object fill factors $b=1$ and $b=0.6, N=30, p=8$.

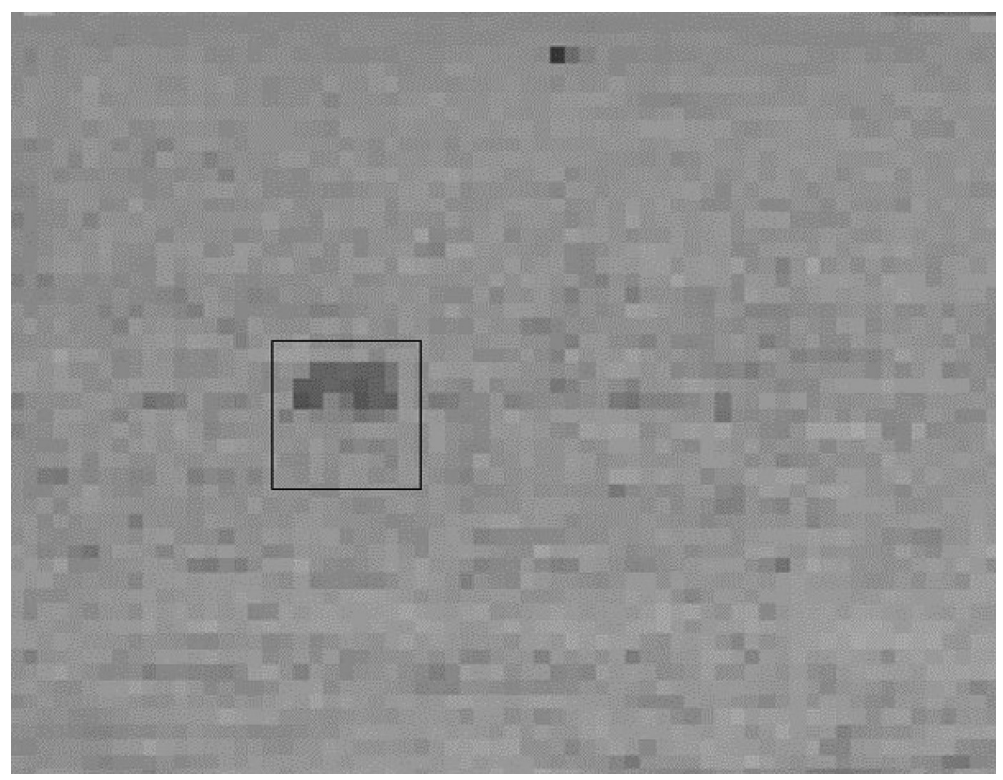

Fig. 13 An image of a floating plastic container 2 (marked observed region is $10 \times 10$ pixels).

pixel 2). The second experimental results show that the MSpMD detection performance significantly outperforms the HMSD at any object fill factor.

\section{Conclusion}

In this paper, we have proposed MSpMD for subpixel targets in the presence of the Gaussian unstructured background and noise. We derived the GLRT for the case where the part of the background spectra is estimated under the null and the alternative hypotheses. The proposed detector modifies the classical SpMD by adding the corrective term proportional to the energy 


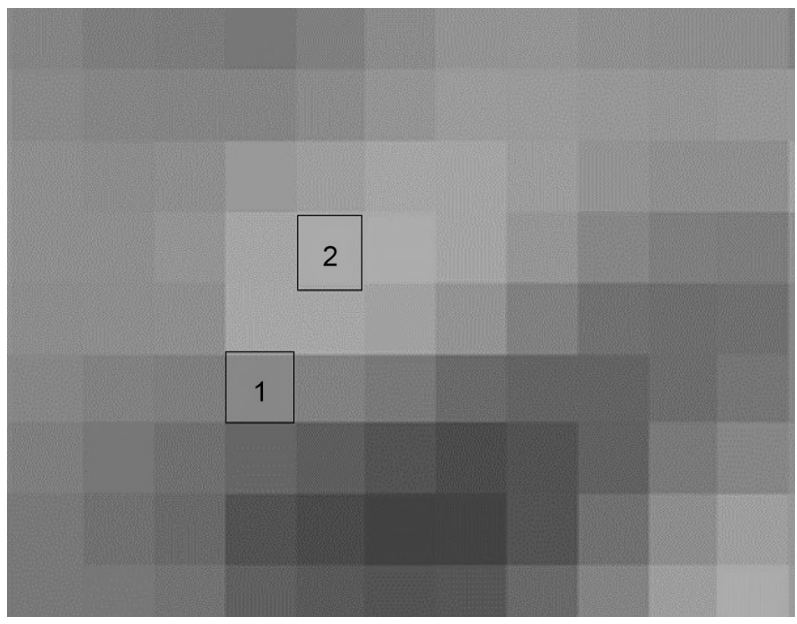

Fig. 14 The observed region (marked in Fig. 13) with size $7 \times 10$ pixels and two marked pixels with different object fill factors $b$.

variation (under null and alternative statistical hypotheses) of the spectrum at frequencies more than the maximum object frequency. Numerical simulations and two series of experiments conducted on the real dataset show that the new detector MSpMD considerably outperforms the SpMD and the recently proposed HMSD. The MSpMD, HMSD, and SpMD efficiencies depend on contrast between mean and variance of the reflections from the background and object, the object fill factor, ratio channel noise variance-background variance, and number of pixels in received signal. However, only MSpMD is sensitive to the spectrum shape contrast of the received signal under null and alternative hypotheses. The MSpMD was demonstrated as an effective way of subpixel target detection in the case of barely discernible small floating objects.

\section{References}

1. D. Manolakis and G. Shaw, "Detection algorithms for hyperspectral imaging applications," IEEE Sign. Proc. Mag. 19(1), 29-43 (2002).

2. A. Borghgraef et al., "An evaluation of pixel-based methods for the detection of floating objects on the sea surface," EURASIP J. Adv. Signal Process. 2010(1), 978451 (2010).

3. J. Chen and I. Reed, "A detection algorithm for optical targets in clutter," IEEE Trans. Aerosp. Electron. Syst. AES-23(1), 46-59, (1987).

4. A. Margalit, I. Reed, and R. Gagliardi, "Adaptive optical target detection using correlated images," IEEE Trans. Aerosp. Electron. Syst. AES-21(3), 394-405, (1985).

5. I.S. Reed and X. Yu, "Adaptive multi-band CFAR detection of an optical pattern with unknown spectral distribution," IEEE Trans. Acoustic Speech Signal Process. 38(10), 1760-1770 (1990).

6. B. Porat and B. Friedlander, "A frequency domain algorithm to multiframe detection and estimation of dim targets," IEEE Trans. Pattern Analysis Mach. Intell. 12(4), 398-401 (1990).

7. K. Garlach, M. Steiner, and F. Lin, "Detection of spatially distributed targets in white noise," IEEE Signal Process. Lett. 4(7), 128-200 (1997).

8. I. S. Reed, J. D. Mallet, and L. E. Brennan, "Rapid convergence rate in adaptive arrays," IEEE Trans. Aerosp. Electron. Syst. AES-10(6), 853-863 (1974).

9. E. J. Kelly, “An adaptive detection algorithm," IEEE Trans. Aerosp. Electron. Syst. AES22(2), 115-127 (1986).

10. S. Kraut and L. Scharf, "The CFAR adaptive subspace detectors is a scale-invariant GLRT," IEEE Trans. Signal Process. 47(9), 2538-2541 (1999).

11. S. Kraut, L. Scharf, and L. McWorther, "Adaptive subspace detectors," IEEE Trans. Signal Process. 49(1), 1-16 (2001). 
12. F. Vincent, O. Besson, and C. Richard, "Matched subspace detection with hypothesis dependent noise power," IEEE Trans. Signal Process. 56(11), 5713-5718 (2008).

13. V. Golikov et al., "Asymptotically optimum quadratic detection on the case of subpixel targets," IEICE Trans. Fund. E94-(9), 1786-1792 (2011).

14. L. Scharf, Statistical Signal processing: Detection, Estimation and Time Series Analysis, Addison-Wesley, Reading, Massachusetts (1991).

15. S. M. Kay, Fundamentals of Statistical Signal Processing: Detection Theory, Prentice-Hall PTR (1998).

16. H.-C. So et al., "On four suboptimal quadratic detectors for random signals," IEICE Trans. Commun. E88-B(12), 4527-4533 (2005).

17. J. Broadwater and R. Chellappa, "Hybrid detectors for subpixel targets," IEEE Trans. Pattern Anal. Mach. Intell. 29(11), 1891-1903 (2007).

18. L. Zhang, B. Du, and Y. Zhong, "Hybrid detectors based on selective endmembers," IEEE Trans. Geosci. Remote Sens. 48(6), 2633-2646 (2010).

19. J. W. Boardman and F. A. Kruse, "Analysis of imaging spectrometer data using Ndimensional geometry and a mixture-tuned matched filtering (MTMF) approach," IEEE Trans. Geosci. Remote Sens. 49(11), 4138-4152 (2011).

20. J. J. Mitchell and N. F. Glenn, "Subpixel abundance estimates in mixture-tuned matched filtering classifications of leafy spurge," Int. J. Remote Sens. 30(23), 6099-6119 (2009).

21. I. Dópido et al., "A quantitative and comparative assessment of unmixing-based feature extraction techniques for hyperspectral image classification," IEEE J. Sel. Top. Appl. Earth Observ. Remote Sens. 5(2), 421-435 (2012).

22. H.C. So, W.-K. Ma, and Y.T. Chan, "Detection of random signals via spectrum matching," IEEE Trans. Aerosp. Electron. Syst. 38(1), 301-307 (2002).

23. Z. Wang and J.-H. Xue, "The matched subspace detector with interaction effects," Pattern Recognit. 68(7), 24-37 (2017).

24. Z. Wang and J.-H. Xue, "Matched shrunken subspace detectors for hyperspectral target detection," Neurocomputing 272(10), 226-236 (2018).

25. Z. Wang et al., "Matched shrunken cone detector (MSCD): Bayesian derivations and case studies for hyperspectral target detection," IEEE Trans. Image Process. 26(11), 5447-5461 (2017).

26. X. Yang et al., "MSDH: matched subspace detector with heterogeneous noise," Pattern Recognit. Lett. 125(7), 701-707 (2019).

27. B. Du et al., "A hypothesis independent subpixel target detector for hyperspectral images," Signal Process. 110(5), 244-249 (2015).

Victor Golikov (PhD 1976, Dr. Sc. 1992) is a professor at the Autonomous University of Carmen, Mexico. He received his MS degree in radiophysics from the National University of Kharkov in 1970 and his $\mathrm{PhD}$ in radar systems from the National Radio-Electronic University of Kharkov in 1976. He is the author of more than 60 journal papers. His current research interests include remote optoelectronic systems.

Oleg Samovarov ( $\mathrm{PhD} 2015)$ is a scientific secretary at Ivannikov Institute for System Programming of the Russian Academy of Sciences and head of the Science Laboratory in Plekhanov Russian University of Economics. He received his $\mathrm{PhD}$ in technical sciences in 2015. He is the author of more than 13 international scientific and conference papers. His current research interests include key technologies for private and hybrid clouds creation, highperformance computing, and other services in clouds and big data ecosystem.

Evgeniy Zhilyakov (PhD 1978, Dr. Sc. 1994) is a professor at Belgorod State National Research University, Russia. He is the author of more than 50 journal papers. His current research interests include information technology and computer systems.

José L. Rullán-Lara graduated in electromechanical engineering from the Universidad Autónoma del Carmen (UNACAR) in 2004, received his MSc in electronic from CENIDET, Mexico, in 2006, and received his $\mathrm{PhD}$ from the Université de Technologie de Compiègne, 
France, in 2011. Since 2006, he has been with the Faculty of Engineering at UNACAR. His current research interests include unmanned vehicles, nonlinear control, and fault detection.

Hussain Alazki is a professor and researcher of Autonomous University of Carmen, Mechatronic Department, Mexico. He received his postdoc from the University National Autonomous of Mexico. He received his PhD in automatic control from the Centre for Research and Advanced Studies of National Polytechnic Institute (CINVESTAV). He received his bachelor's degree in electronic engineering from Tishreen University, 2001, a diploma in communication engineering in 2002, and his MSc degree in telecommunication engineering from Tishreen University, Faculty of Electrical and Mechanical Engineering in 2007. His research interests include autonomous systems, robust nonlinear control, identification and stochastic models. 